\title{
Conductas de ciberadicción y experiencias de cyberbullying entre adolescentes
}

\author{
Pilar Arnaiz ${ }^{1}$, Fuensanta Cerezo ${ }^{2 *}$, Ana M. Giménez ${ }^{1}$ y Javier J. Maquilón ${ }^{2}$ \\ ${ }^{1}$ Facultad de Educación, Universidad de Murcia (España). \\ ${ }^{2}$ Facultad de Psicología, Universidad de Murcia (España).
}

\begin{abstract}
Resumen: La relación de los jóvenes con las tecnologías plantea diversos riesgos como la ciberadicción y el cyberbullying. Este estudio analiza el consumo que una muestra de adolescentes hace del móvil y del ordenador, de conductas de ciberadicción, y de bullying y cyberbullying, teniendo en cuenta su relación con el sexo y el nivel educativo. También se relaciona el consumo con la supervisión familiar durante la conexión a la red. Se aplicó un cuestionario autoinformado a 1353 adolescentes escolares de Educación Secundaria y Bachillerato, de un rango de edad de 12-21 años $(M=14.8$; $D T=1.62 ; 52.8 \%$ varones). Los resultados indican que el consumo medio del móvil y del ordenador se encuentra entre 1-2 horas al día. Casi un 13\% se encuentra en situación de ciberadicción y el $32 \%$ en situación de riesgo. Se aprecian diferencias por sexo y nivel educativo. Se constata un mayor nivel de incidencia del bullying (12\%) frente al cyberbullying (7.7\%), así como una considerable asociación entre estas conductas y el consumo de medios. La supervisión familiar actúa como factor de protección. Estos resultados plantean la necesidad de alentar a adolescentes y educadores sobre la importancia de educar en los riesgos del abuso de las tecnologías.

Palabras clave: ciberadicción; bullying; cyberbullying; supervisión parental;
\end{abstract} educación secundaria.

\section{Introducción}

Las Tecnologías de la Información y la Comunicación (en adelante, TIC) forman parte del quehacer diario de niños y adolescentes, su uso cotidiano es cada vez más precoz. Las investigaciones de los últimos años vienen alertando de los numerosos riesgos que se derivan del interés de los menores hacia las TIC (Garmendia, Garitaonandia, Martínez y Casado, 2012), destacando su influencia en el desarrollo y bienestar psicológico (Devine y Lloyd, 2012), e incluso con el clima familiar.

La mayor parte de las investigaciones sobre el consumo de medios se ha situado en la etapa de la adolescencia, obviando el colectivo de niños de edades tempranas (8-11 años) que accede cada vez con más asiduidad a las mismas. Estudios a nivel europeo ratifican esta situación poniéndose énfasis tanto en el temprano acceso a las TIC por los menores como el consumo de las mismas cada vez más masivo y dilatado en el tiempo (Ólafsson, Livingstone y Haddon, 2013). En España, estudios como el de Garmendia et al. (2012) encuentran que el 56\% de los menores entre los 9-16 años accede a internet todos o casi todos los días, y un $82 \%$ en la franja de los 15-16 años con una media diaria de 71 minutos. Entre las principales actividades que desarrollan durante la conexión destacan la realización de tareas escolares $(83 \%)$, jugar $(80 \%)$ y el visionado/descarga de vídeos $(78 \%)$. Datos más actuales, indican que los escolares entre los 10-17 años acceden todos (58.8\%) o casi todos los días a

* Dirección para correspondencia [Correspondence address]: Fuensanta Cerezo. Departamento de Psicología Evolutiva y de la Educación, Facultad de Psicología. Campus de Espinardo. Espinardo CP. 30100. Murcia (España). E-mail: fcerezo@um.es

\begin{abstract}
Title: Online addiction behaviors and cyberbullying among adolescents. Abstract: The relationship between young people and technologies implies some risks like online addiction and cyberbullying. This study analyses the use of mobile phones and computers in a sample of adolescents, their online addiction behaviours, and bullying and cyberbullying experiences considering the influence of gender and school level. Parental control during Internet use is also considered. Using a self-report questionnaire, 1353 secondary and high school adolescents between 12 -21 years-old participated $(M=14.8 ; S D=1.62 ; 52.8 \%$ boys $)$. Results show an average of $1-2$ hours daily use of mobile phone and computers. Around $13 \%$ of students report online addictions behaviors and $32 \%$ are in risk of, with differences by gender and school level. Results indicate more involvement in traditional bullying $(12 \%)$ than in cyberbullying $(7.7 \%)$, and a significant association between both behaviors and technologies use. Besides, parental mediation acts as protective factor. In conclusion, this study underlines the need to teach young people and educators about risks regarding excessive use of technology.

Keywords: online addiction; bullying; cyberbullying; parental mediation; secondary education.
\end{abstract}

internet $(25.7 \%)$ entre 1 y 2 horas diarias (41.9\%), 2-3 ho$\mathrm{ras} /$ día $(22.3 \%)$ o más de tres horas/día (22.5\%) (Ministerio del Interior, 2014).

Entre los usos que se le presta a las TIC, destaca la profusión del uso de las redes sociales entre la población adolescente (Rial, Gómez, Braña y Valera, 2014), con una mayor presencia de chicas como usuarias. Se encuentran diferencias por sexo en los usos que se hacen de las TIC, así entre las mujeres es más frecuente su empleo para la creación y mantenimiento de las amistades, frente a los varones que recurren a ellas para reforzar su autoestima y cubrir facetas emocionales (Colás, González y de Pablos, 2013).

Es evidente que el mundo virtual ofrece a los menores un espacio de infinitas posibilidades pero también de numerosos riesgos, entre los que cabe destacar la adicción a las mismas o ciberadicción y el uso malévolo como el cyberbullying. En primer lugar, cuando su consumo se torna excesivo, se produce un estado de dependencia de las mismas que puede convertirse en una adicción patológica, restando libertad a la persona al estrecharse su campo de conciencia y amplitud de intereses (Labrador, Villandangos, Crespo y Becoña, 2013). Aunque todavía no existe consenso para determinar qué supone un uso adictivo, existen algunos criterios comunes para su detección y diagnóstico como son: la dedicación temporal excesiva, pérdida de control y tolerancia, síntomas de abstinencia como ansiedad e irritabilidad y la clara interferencia con las actividades de la vida cotidiana (Echeburúa y Corral, 2010).

Estudios recientes ponen de manifiesto la extensión de la adicción a internet, a las TIC y los usos problemáticos de la red entre la población joven (Cao, Sun, Wan, Hao y Tao, 2011; Carbonell, Fúster, Chamarro y Oberst, 2012; Gómes y Sendín, 2014; Labrador et al., 2013; Pedrero, Rodríguez y 
Ruiz, 2012), alertando de las múltiples consecuencias negativas como pueden ser: el consumo de sustancias, depresión, trastornos del sueño e inmunológicos, malestar psicológico, agresividad, aislamiento, problemas de adaptación social y reducción del rendimiento académico. También, análisis centrados en el tiempo de uso de las redes sociales y las consecuencias para la salud mental de los adolescentes señalan la existencia de problemas externalizantes entre los consumidores más asiduos (Rodríguez y Fernández, 2014).

Otros riesgos a los que se exponen los jóvenes provienen de la utilización de las TIC para violentar, vejar y humillar a otros. Las tecnologías ofrecen un espacio idóneo para acosar a otros aprovechando la multiplicidad de medios, la sensación de impunidad y de invisibilidad ante los adultos. Precisamente es en este espacio virtual donde se produce el gyberbullying debido al abuso de poder que una persona o varias ejercen contra una víctima a través de los medios electrónicos (Slonje, Smith y Frisén, 2013). Son numerosas las investigaciones que corroboran la relación de este hecho con el bullying tradicional (Schneider, O’Donnell, Stueve y Coulter, 2012; Smith et al., 2008; Vandebosch y Van Cleemput, 2009), aunque esta forma de acoso presenta unas características novedosas que lo dotan de entidad propia como son: el anonimato, una audiencia más amplia y universal y la mayor permanencia en el tiempo de las agresiones (Garaigordobil y Martínez, 2014).

La prevalencia del cyberbullying ha ido en aumento desde los inicios de su investigación hasta revisiones actuales, encontrando, según los estudios, un índice de victimización entre el 5-20\% y de agresión entre el 10-40\% (Álvarez et al., 2011; Garaigordobil, 2011; Giménez, Maquilón y Arnaiz, 2014; León, Felipe, Fajardo y Gómez, 2012; Ortega, Calmaestra y Mora-Merchán, 2008).

Las investigaciones sobre cyberbullying han tenido en cuenta variables relacionadas como: la existencia de diferencias por género, curso, nacionalidad e incluso en relación con el consumo y usos de las TIC. Sobre el género, los resultados son contradictorios pues mientras algunos autores no encuentran diferencias (Álvarez et al., 2011; Smith et al., 2008), otros observan mayor tendencia de las chicas a ser cibervíctimas y de chicos a ser ciberagresores (Ortega, Calmaestra y Mora-Merchán, 2008; Patchin y Hinduja, 2012; Schneider et al., 2012). Respecto a la edad, se aprecia mayor consenso entre autores, siendo los primeros años de la adolescencia (12-15 años) el momento de mayor incidencia, y observando una reducción conforme aumenta la edad (Slonje et al., 2013; Tokunaga, 2010). Otros estudios relacionan el uso diario de las redes sociales y la ciberagresión (León, Felipe, Fajardo y Gómez, 2012). Sin embargo, constatan que los menores que hacen un mayor consumo de las TIC se muestran más proclives a convertirse en víctimas y agresores del ciberacoso (Erdur-Baker, 2010).

De todo lo dicho, es de esperar que las consecuencias que se derivan de la implicación en cyberbullying afecten a todos sus implicados y, en mayor grado, a las víctimas del mismo ya que pueden llegar a sufrir trastornos somáticos, bajo bienestar psicológico y otros síntomas como depresión, ira, ansiedad, conductas de riesgo, miedo, deterioro de la autoestima $y$, en los peores casos, conductas suicidas consumadas (Devine y Lloyd, 2012; Garaigordobil, 2011).

Frente a los riesgos de internet comentados, la supervisión parental durante el tiempo de conexión a las tecnologías $y$, concretamente a internet, se erige en factor importante de protección ante los riesgos del ciberespacio (Leung y Lee, 2012). No obstante, cabe indicar que todavía no es una práctica demasiado extendida y que se centra sobre todo en la restricción del tiempo de uso (Martínez, Cortés, Medrano y Apodaca, 2014).

La revisión precedente pone de manifiesto que las TIC sitúan a los menores en un escenario de riesgos donde las conductas de adicción y el cyberbullying son preocupantes. Aunque la prevalencia del acoso en la red entre los adolescentes españoles aún no es tan alta como en otros países europeos, América o Canadá, investigaciones llevadas a cabo en la Región de Murcia confirman su existencia (Giménez, Maquilón y Arnaiz, 2014). Por ello, este trabajo analiza las características y variables influyentes en el cyberbullying entre adolescentes españoles planteando los siguientes objetivos: a) analizar la accesibilidad de los menores a las TIC y su consumo diario, b) analizar las conductas de adicción a las TIC y su relación con conductas de bullying y cyberbullying, y c) analizar la supervisión que los padres realizan del uso de las TIC por parte de sus hijos y la relación con la implicación en bullying y cyberbullying.

\section{Método}

\section{Participantes}

En esta investigación han participado un total de 1353 escolares de educación secundaria de 21 centros de la Región de Murcia (60.6\% de titularidad pública, 24.7\% concertada y $14.7 \%$ privada no concertada), distribuidos entre los cuatro cursos de Educación Secundaria Obligatoria (ESO), siendo: $1^{\circ} \mathrm{ESO}(n=248 ; 18.3 \%), 2^{\circ} \mathrm{ESO}(n=242 ; 17.9 \%)$, $3^{\circ} \mathrm{ESO}(n=356 ; 26.3 \%), 4^{\circ} \mathrm{ESO}(n=323 ; 23.9 \%)$, y primer curso de Bachillerato $(n=184 ; 13.6 \%)$ de entre 12 y 21 años $(M=14.8, D T=1.62),(47.2 \%$ mujeres $)$. El $88.2 \%$ estudiantes españoles y el resto de otras nacionales (Marruecos, Argelia y Europa del Este).

El muestreo realizado es de tipo estratificado siendo la unidad primaria la titularidad de los centros y la unidad secundaria el nivel educativo (ESO y bachillerato). Realizado el cálculo de la representatividad de la muestra, con un nivel de confianza del $99 \%$ y un error muestral del $5 \%$, se obtuvo que debieran encuestarse a un mínimo de 660 estudiantes, por lo que la muestra final encuestada resultó representativa de la provincia de Murcia. 


\section{Variables e Instrumentos}

Para la evaluación del uso de las TIC y de las conductas de acoso se utilizó el cuestionario autoinformado "Cyberbull" elaborado ad hoc (Anexo I). El diseño de su estructura y contenido partió de la revisión del DAPHNE Questionnaire (Calmaestra, 2011). Mediante método Delphi de validación por juicio de expertos, y con dos rondas de evaluación, se calculó la validez de contenido. Este proceso permitió la configuración de la estructura final de instrumento dispuesto por cinco dimensiones con un total de 27 preguntas: a) relación de los menores con las TIC, b) experiencias de bullying en el último mes, c) experiencias de cyberbullying en el último mes, d) estrategias de afrontamiento ante el cyberbullying, y d) espectadores ante la violencia escolar. Al final del mismo, se incluyen las variables sociodemográficas: edad, sexo, nivel educativo, nacionalidad, participación en programas de apoyo educativo y nivel educativo de los padres. En este estudio se incluyen las variables que conforman las tres primeras dimensiones.

Algunos ítems del cuestionario se acompañan de una escala dicotómica Sí/No, que da paso a otros de opción múltiple o tipo Likert con cinco opciones de respuesta donde: $1=$ nunca y $5=$ siempre. En primer lugar, se pregunta a los estudiantes si están en posesión de un teléfono móvil propio y un ordenador, para a continuación solicitar información acerca de la frecuencia de consumo diario $(1=$ menos de 1 hora, $2=1-2$ horas, $3=2-4$ horas, $4=$ más de 4 horas $)$.

Las conductas de ciberadicción se evaluaron a partir de la adaptación del "Cuestionario DANE" (Labrador y Villadangos, 2010), derivando en una escala con nueve ítems relacionados con tres tipos de comportamientos: ansiedad y malestar ante el no uso de las tecnologías (4 ítems); agresividad a partir de discusiones o enfados ante la interrupción de la conexión (2 ítems); y comportamientos que implican cambios de intereses" a partir de la tendencia al aislamiento, cambio en los ritmos de sueño, y preferencia a estar con las TIC frente a la socialización (3 ítems). Siguiendo la clasificación propuesta por Carbonell et al. (2012), se establecieron tres subgrupos: estudiantes sin problemas de ciberadicción, grupo en riesgo, y grupo con conductas adictivas. Los resultados de los análisis de fiabilidad para esta dimensión apuntan que es alta $(a=.82)$.

La supervisión parental se evaluó incluyendo ítems sobre el control y quién ejercía dicha supervisión: padres, abuelos y hermanos mayores, utilizando una escala tipo Likert de cinco puntos siendo $1=$ nunca y $5=$ siempre que arrojó un índice de fiabilidad de $a=.66$.

\section{Procedimiento}

En primer lugar se solicitó a los centros educativos por contacto telefónico su participación para lo cual se les remitió un breve informe sobre los objetivos y alcance de la investigación. Tras recibir el visto bueno por parte de los Equipos Directivos y bajo los criterios éticos de confidencia- lidad y anonimato, se procedió a pasar las encuestas a los alumnos durante el segundo y tercer trimestre del curso académico 2012/2013. El horario convenido fue dentro del lectivo de cada centro con una duración por sesión de 30 minutos. A continuación se procedió al análisis de los cuestionarios, al vaciado y análisis de la información con el programa estadístico SPSS versión 21.0.

\section{Análisis de datos}

Comprobada la normalidad de la muestra e igualdad de varianzas se optó por técnicas paramétricas. Para el análisis descriptivo se utilizaron tablas de frecuencias, porcentajes y de contingencia con el estadístico Chi-cuadrado para medir la asociación entre variables tomando como valor de significación de $p<.05$. Para el contraste de hipótesis de dos grupos se utilizó la $t$ de Student y para más de dos grupos la ANOVA unifactorial con la prueba post hoc de Bonferroni. En los casos en que la $p$ valor es significativa se completa con el estadístico $d$ de Cohen donde $d=0.20$ es considerado un efecto pequeño, $d=.50$ efecto medio, y $d=.80$ efecto grande.

\section{Resultados}

La distribución de la muestra según la implicación en dinámicas de cyberbullying indica que un 7.7\% $(n=104)$ de participación, de los que un $5 \%$ se reconocía como cibervíctima, un $1.5 \%$ como ciberagresores, y un $1.2 \%$ como ciberagresores victimizados. La distribución por curso y titularidad del centro (véase Tabla 1), señala que una mayor proporción se localiza entre los cursos $1^{\circ}, 2^{\circ}$ y $3^{\circ}$ de ESO, disminuyendo en el resto de la muestra. Asimismo se aprecia una mayor proporción de implicados en los centros públicos (56.7\%), frente a concertados $(33.7 \%)$ y privados no concertados $(9.6 \%) ; \chi^{2}(4)=1.13, p=.89$.

Siguiendo el orden de los objetivos propuestos, para dar respuesta al primero de ellos, se analiza la accesibilidad, consumo diario de las TIC y su relación con la implicación en bullying y cyberbullying en el conjunto de los participantes. En cuanto a la accesibilidad a las TIC, el $94.7 \%$ de los adolescentes afirma estar en posesión de un teléfono móvil propio, teniendo el $72.6 \%$ acceso a internet. En el caso del ordenador, un $96.6 \%$ tiene acceso al mismo y el $99.8 \%$ tiene acceso a internet. No se encontró asociación significativa entre la propiedad de móvil y acceso al ordenador con el género. Sí se encontró asociación significativa ente curso y acceso al móvil, $\chi^{2}(2)=9.65, p=.002$ y con el uso del ordenador, $\chi^{2}(2)=5.29, p=.021$.

Respecto al consumo diario del móvil, los resultados indican un mayor consumo por parte de las chicas dedicándole más de cuatro horas diarias (12.5\%), frente al 5\% de los chicos. En la franja de 2 a 4 horas de dedicación, de nuevo las chicas superan a los chicos con un $11.1 \%$ frente al $8 \%$, siendo además tales diferencias significativas, $\chi^{2}(2)=50.53, p<$ .000. En el caso del ordenador, las chicas dedican más de 
cuatro horas diarias $(6.8 \%)$ respecto a los chicos $(4.8 \%)$, aunque las diferencias no resultaron estadísticamente significativas, como tampoco según el nivel educativo y el mayor o menor consumo de ambas tecnologías.

Tabla 1. Implicación en cyberbullying por curso y titularidad del centro educativo.

\begin{tabular}{|c|c|c|c|c|c|}
\hline Curso & Titularidad & $\begin{array}{c}\text { Agresores } \\
n=20 \\
(\%)\end{array}$ & $\begin{array}{c}\text { Víctimas } \\
n=68 \\
(\%)\end{array}$ & $\begin{array}{l}\text { Agresores } \\
\text { victimizado } \\
n=16 \\
(\%)\end{array}$ & $\begin{array}{c}\text { Total } \\
\text { Implicados } \\
\text { Curso } \\
(\%) \\
\end{array}$ \\
\hline \multirow{3}{*}{$1^{\circ} \mathrm{ESO}$} & Pública & - & $9(69.2)$ & $4(30.8)$ & \\
\hline & Concertada & $3(33.3)$ & $5(55.6)$ & 1 (11.1) & \\
\hline & Privada & $2(33.3)$ & $3(50)$ & $1(16.7)$ & \\
\hline \multirow[t]{2}{*}{ Total $1^{\circ}$} & & & & & $28(11.3)$ \\
\hline & Pública & $1(14.3)$ & $6(85.7)$ & - & \\
\hline \multirow[t]{2}{*}{$2^{\circ} \mathrm{ESO}$} & Concertada & $2(40)$ & $2(40)$ & $1(20)$ & \\
\hline & Privada & - & $2(100)$ & - & \\
\hline \multirow[t]{2}{*}{ Total $2^{\circ}$} & & & & & $14(9.9)$ \\
\hline & Pública & $8(33.3)$ & $12(50)$ & 4 (16.7) & \\
\hline \multirow[t]{2}{*}{$3^{\circ} \mathrm{ESO}$} & Concertada & $1(8.3)$ & $9(75)$ & $2(16.7)$ & \\
\hline & Privada & - & $1(100)$ & - & \\
\hline \multirow[t]{2}{*}{ Total $3^{\circ}$} & & & & & $37(10.4)$ \\
\hline & Pública & $1(8.3)$ & $9(75)$ & $2(16.7)$ & \\
\hline \multirow[t]{2}{*}{$4^{\circ} \mathrm{ESO}$} & Concertada & $1(33.3)$ & $2(66.7)$ & - & \\
\hline & Privada & - & - & - & \\
\hline \multirow[t]{2}{*}{ Total $4^{\circ}$} & & & & & 15 (4.6) \\
\hline & Pública & - & $3(100 \%)$ & - & \\
\hline \multirow[t]{2}{*}{$1^{\circ} \mathrm{Bach}$} & Concertada & & $5(83.3 \%)$ & $1(16.7 \%)$ & \\
\hline & Privada & $1(100 \%)$ & - & - & \\
\hline $\begin{array}{l}\text { Total } 1 \\
\text { Bachiller } \\
\end{array}$ & & & & & $9(5)$ \\
\hline
\end{tabular}

El análisis de diferencias entre los implicados en ambas dinámicas de acoso y el consumo diario de las TIC, tomando como variable de agrupación los implicados en agresión y ciberagresión (agresores y agresores victimizados) y en victimización y cibervictimización, los resultados indican que aquellos que ejercen el acoso desde las formas tradicional, $t(1348)=-2.08, p=.038, d=.11$, como utilizando el móvil y el ordenador, $t(1348)=-2.82, p=.005, d=.22$, son los que aseguran hacer un consumo mayor de las tecnologías. Tales diferencias no son significativas entre el grupo de los no implicados e implicados como víctimas y cibervíctimas.

En el segundo objetivo se han analizado las conductas de uso problemático y de adicción a las TIC. El factor de ciberadicción generado a partir del cómputo de las nueve conductas examinadas, permite distinguir entre sujetos con problemas, sin problemas y en riesgo (Carbonell et al, 2012). Los resultados indican que un $55.4 \%$ de los encuestados se sitúa en el grupo categorizado como "sin problemas", un $32.1 \%$ "en riesgo", y un restante $12.5 \%$ de escolares "con problemas" de posible uso problemático y adictivo de las
TIC. Entre éstas, la conducta referida a la ansiedad por no poder utilizar el ordenador es la que presenta valores más elevados $(M=2.67, D T=1.25)$, seguida de la ansiedad sin el móvil $(M=2.49, D T=1.33)$. Entre las conductas con valores medios más bajos aparecen la preferencia a estar con las TIC en vez de con la familia y amigos $(M=1.33, D T=.68)$, o la discusión con la familia por el tiempo de dedicación a las tecnologías $(M=1.74, D T=1.00)$.

En cuanto a las diferencias por sexo, las chicas sufren mayor grado de malestar cuando no tienen acceso al móvil, $t(1351)=-6.38, p<.000, d=1.11$, ansiedad sin móvil, $t(1351)=-7.11, p<.000, d=1.38$, cambio en los ritmos de sueño por prolongar el tiempo de conexión, $t(1351)=-2.56$, $p=.010, d=.18$, y mayores episodios de discusión con los padres cuando se les interrumpe mientras acceden a internet, $t(1351)=-2.23, p=.026, d=.14$. En cambio, son los chicos quienes significativamente más se encierran en su habitación para usar las TIC, $t(1351)=2.46, p=.014, d=.17$.

Según el nivel educativo, se encuentran diferencias significativas entre los cuatro cursos de la ESO y primer curso de bachillerato en las variables de cambio en los ritmos de sueño, $F(4,1348)=20.50, p<.000$, ansiedad sin móvil, $F(4$, $1348)=2.96, p=.019$, ansiedad sin ordenador $F(4,1348)=$ 2.74, $p=.028$, encierro o aislamiento en la habitación, $F(4$, $1347)=6.30, p<.000$, y discusión por el tiempo de conexión, $F(4,1348)=2.88, p=.022$.

$\mathrm{El}$ análisis de la relación entre bullying y cyberbullying y el factor global de ciberadicción señala que aquellos que afirman estar implicados en dinámicas de bullying tradicional $(M$ $=20.67, D T=6.86)$ presentan mayor tendencia al uso problemático y adictivo a las tecnologías que aquellos que no participan de este tipo de acoso $(M=18.59, D T=6.72)$; $t(1349)=-3.69, p<.000 ; d=.37$. De forma similar sucede en el caso del cyberbullying, siendo los implicados en cualquiera de los roles los que mayores muestras dan de conductas problemáticas de uso de las TIC $(M=21.14, D T=7.32)$ frente a los que no participan $(M=18.65, D T=6.69) ; t$ $(1349)=-3.63, p<.000, d=.36)$.

La comparación entre víctimas y agresores en bullying con los no implicados (Tabla 2) indica que las víctimas sienten mayor malestar sin el móvil $(M=2.02, D T=1.13)$, que prefieren estar utilizando las TIC en lugar de establecer relaciones con la familia y amigos $(M=1.48, D T=0.91)$, y que discuten más con la familia por su dedicación continuada a las tecnologías $(M=1.93, D T=1.09)$ que los no victimizados. Por su parte, los agresores son los que más conductas de uso problemático y adictivo a las TIC manifiestan $(M=$ $23.35, D T=6.85)$ sobre los no agresores $(M=20.41, D T=$ 7.37), y además de forma significativa en todos los casos excepto en la preferencia a utilizar las TIC. 
Tabla 2. Conductas de ciberadicción, agresión y victimización en bullying.

\begin{tabular}{|c|c|c|c|c|c|}
\hline & No & Sí & $t$ & $p$ & $d$ \\
\hline \multicolumn{6}{|l|}{ Victimización } \\
\hline Malestar sin móvil & $1.83(1.08)$ & $2.02(1.13)$ & -1.775 & .076 & ns \\
\hline Malestar sin ordenador & $2.18(1.19)$ & $2.57(1.20)$ & -3.584 & .000 & .35 \\
\hline Discusión por interrumpir & $1.99(1.17)$ & $2.19(1.26)$ & -1.833 & .067 & ns \\
\hline Cambios ritmo del sueño & $2.17(1.28)$ & $2.19(1.34)$ & -.200 & .842 & ns \\
\hline Ansiedad sin móvil & $2.50(1.33)$ & $2.40(1.29)$ & .791 & .429 & ns \\
\hline Ansiedad sin ordenador & $2.64(1.24)$ & $2.86(1.29)$ & -1.910 & .056 & ns \\
\hline Aislamiento & $2.36(1.31)$ & $2.52(1.42)$ & -1.279 & .201 & ns \\
\hline Preferencia de las TIC & $1.31(0.66)$ & $1.48(0.91)$ & -2.668 & .008 & .19 \\
\hline Discusión por el tiempo & $1.72(0.99)$ & $1.93(1.09)$ & -2.223 & .026 & .13 \\
\hline \multicolumn{6}{|l|}{ Agresión } \\
\hline Malestar sin móvil & $1.83(1.07)$ & $2.24(1.28)$ & -2.717 & .007 & .20 \\
\hline Malestar sin ordenador & $2.20(1.20)$ & $2.64(1.24)$ & -2.639 & .008 & .19 \\
\hline Discusión por interrumpir & $2.00(1.18)$ & $2.38(1.23)$ & -2.269 & .023 & .14 \\
\hline Cambios ritmo del sueño & $2.15(1.28)$ & $2.70(1.41)$ & -3.037 & .002 & .25 \\
\hline Ansiedad sin móvil & $2.47(1.33)$ & $2.87(1.33)$ & -2.116 & .035 & .12 \\
\hline Ansiedad sin ordenador & $2.65(1.25)$ & $3.08(1.24)$ & -2.455 & .014 & .16 \\
\hline Aislamiento & $2.35(1.32)$ & $2.91(1.50)$ & -2.974 & .003 & .24 \\
\hline Preferencia de las TIC & $1.32(0.68)$ & $1.43(0.84)$ & -1.150 & .250 & ns \\
\hline Discusión por el tiempo & $1.73(0.99)$ & $2.09(1.23)$ & -2.608 & .009 & .19 \\
\hline
\end{tabular}

En el caso del cyberbullying los resultados son muy similares a los reportados anteriormente (Tabla 3). De nuevo los ciberagresores son los que muestran más conductas adictivas hacia las TIC $(M=23.25, D T=18.72)$ en comparación con los no implicados $(M=18.72, D T=6.70) ; t(1349)=-3.98, p$ $<.000, d=.43$, y además de forma significativa en todas las conductas analizadas a excepción de la ansiedad sin el orde- nador y la preferencia a las TIC. Las cibervíctimas $(M=$ $20.50, D T=6.96)$ también superan a las que no lo son $(M=$ $18.73, D T=6.74)$ en todas las conductas analizadas, $t(1349)$ $=-2.32, p<.020, d=.15$, aunque las diferencias sólo resultan significativas en un mayor malestar sin el ordenador $(p=$ .014), y la discusión por el tiempo de uso respecto a las que posicionan como no implicados $(p=.012)$.

Tabla 3. Conductas de ciberadicción, agresión y victimización en cyberbullying.

\begin{tabular}{|c|c|c|c|c|c|}
\hline & No & Sí & $t$ & $p$ & $d$ \\
\hline \multicolumn{6}{|l|}{ Cibervictimización } \\
\hline Malestar sin móvil & $1.84(1.08)$ & $2.00(1.15)$ & -1.311 & .190 & ns \\
\hline Malestar sin ordenador & $2.20(1.19)$ & $2.50(1.27)$ & -2.243 & .025 & .14 \\
\hline Discusión por interrumpir & $1.99(1.18)$ & $2.27(1.27)$ & -2.061 & .039 & .12 \\
\hline Cambios ritmo del sueño & $2.16(1.28)$ & $2.42(1.40)$ & -1.792 & .753 & ns \\
\hline Ansiedad sin móvil & $2.48(1.33)$ & $2.60(1.37)$ & -.753 & .451 & ns \\
\hline Ansiedad sin ordenador & $2.65(1.25)$ & $2.89(1.30)$ & -1.744 & .081 & ns \\
\hline Aislamiento & $2.36(1.32)$ & $2.56(1.44)$ & -1.311 & .190 & ns \\
\hline Preferencia de las TIC & $1.32(.68)$ & $1.43(.83)$ & -1.392 & .164 & ns \\
\hline Discusión por el tiempo & $1.74(1.00)$ & $1.83(.99)$ & -.854 & .393 & ns \\
\hline \multicolumn{6}{|l|}{ Ciberagresión } \\
\hline Malestar sin móvil & $1.83(1.07)$ & $2.47(1.42)$ & -3.508 & .000 & .33 \\
\hline Malestar sin ordenador & $2.20(1.19)$ & $2.78(1.44)$ & -2.854 & .004 & .22 \\
\hline Discusión por interrumpir & $2.00(1.18)$ & $2.56(1.27)$ & -2.777 & .006 & .21 \\
\hline Cambios ritmo del sueño & $2.15(1.27)$ & $2.92(1.54)$ & -3.526 & .000 & .34 \\
\hline Ansiedad sin móvil & $2.48(1.33)$ & $2.92(1.36)$ & -1.955 & .051 & .10 \\
\hline Ansiedad sin ordenador & $2.65(1.25)$ & $3.03(1.25)$ & -1.776 & .076 & ns \\
\hline Aislamiento & $2.36(1.32)$ & $3.05(1.41)$ & -3.126 & .002 & .27 \\
\hline Preferencia de las TIC & $1.33(0.69)$ & $1.36(0.59)$ & -.298 & .766 & ns \\
\hline Discusión por el tiempo & $1.73(0.99)$ & $2.17(1.28)$ & -2.575 & .010 & .18 \\
\hline
\end{tabular}

Profundizando en la búsqueda de diferencias intergrupos por roles de participación en ambas dinámicas de acoso entre iguales, aparecen diferencias entre roles en bullying para las variables relacionadas con el descontrol en los ritmos de sueño, $F(2,159)=4.06, p=.019$, y en ansiedad por no acceder al móvil, $F(2,159)=3.60, p=.029$. En cyberbullying, las diferencias entre los tres roles de implica- ción aparecen únicamente en la variable referida a malestar sin el móvil, $F(2,101)=3.63, p=.030$.

También se analiza si agresores y víctimas de cyberbullying difieren tanto en el factor global de ciberadicción como en cada una de las nueve conductas examinadas. En el primer caso, los ciberagresores puntúan con un valor más elevado en el factor de ciberadicción que las cibervíctimas, 
$t(86)=-2.07, p=.041, d=.46 . \mathrm{Al}$ considerar las distintas conductas, aparecen diferencias significativas en el descontrol de los ritmos del sueño más común entre los acosadores $(M=2.05, D T=.94)$ frente a las cibervíctimas $(M=1.59$, $D T=.87) ; t(86)=-2.05, p=.043, d=.45$, como también en la discusión por interrumpir la conexión a internet de nuevo más propio de los agresores $(M=1.70, D T=.92)$ que de las víctimas $(M=1.25, D T=.61) ; t(86)=-2.56, p=.012, d=$ .71 .

Por último, para dar respuesta al tercer objetivo, se realizaron los análisis descriptivos y contingencias sobre la supervisión por parte de distintos familiares (padres, hermanos mayores y abuelos) durante la conexión a la red por los hijos. De la muestra encuestada, sólo un 27.1\% $(n=367)$ reconoce ser supervisado por algún adulto. Dentro de la unidad familiar son los padres los que se atribuyen la labor de supervisión $(M=3.13, D T=1.35)$, por delante de los hermanos mayores $(M=2.03, D T=1.30)$, y de los abuelos $(M=1.22$, $D T=.62)$. El análisis por sexo apunta que la supervisión familiar, ya sea ejercida por padres, hermanos o abuelos está más dirigida a las chicas $(M=1.92, D T=.57)$ que a los chi$\cos (M=1.82, D T=.59) ; t(365)=-2.51, p=.013, d=.26$. También se observa que la familia supervisa más a los hijos que están cursando ESO $(M=1.90, D T=.59)$ que bachillerato $(M=1.61, D T=.41) ; t(365)=2.54, p=.011, d=.27$.

$\mathrm{Al}$ considerar la participación en bullying y cyberbullying, se observa que aquellos menores que afirman estar implicados en acoso escolar tradicional son más supervisados por la familia $(M=5.79, D T=1.50)$ que los no implicados $(M=$ $5.54, D T=1.41)$. Sin embargo, en el caso del cyberbullying sucede todo lo contrario, pues los que sí están implicados muestran niveles menos elevados de control familiar/parental $(M=5.36, D T=1.26)$ que los que no están implicados $(M=5.61, D T=1.45)$, aunque para ninguna de las dos dinámicas de agresión las diferencias entre ambos grupos fueron significativas. Comparando a agresores y víctimas de acoso escolar tradicional, son las víctimas las que afirman ser más supervisadas durante su conexión a la red $(M=5.95, D T=1.52)$ frente a los agresores $(M=4.90, D T$ $=1.10) ; t(46)=2.03, p=.048, d=0.61$, hecho que se repite entre las cibervíctimas, $(M=5.47, D T=1.36)$ y ciberagresores $(M=5.20, D T=1.30)$, aunque en este caso las diferencias no son significativas.

\section{Discusión y conclusiones}

En el presente estudio se ha analizado la accesibilidad de los menores a la telefonía móvil y a internet, su consumo diario y los riesgos asociados a los mismos como son las conductas de uso problemático y adictivo, y el cyberbullying.

Los datos corroboran que tanto el uso del móvil como del ordenador está ampliamente extendido en la muestra adolescente estudiada empleando entre una y dos horas de media al día junto a un cinco por ciento de chicos y un trece por ciento de chicas que dedican un tiempo diario superior a cuatro horas, resultados comparables a los encontrados por
Viñas y González (2010). Aunque otros estudios con muestras españolas señalan un consumo diario y semanal inferior (Labrador et al., 2013; Labrador y Villadangos, 2010; Martínez et al., 2014), podría ser debido a que la naturalidad con que los jóvenes utilizan el teléfono móvil y el ordenador les lleva a infravalorar el consumo real que realizan de estas tecnologías.

Respecto a la incidencia de las experiencias de yybrbullying entre los escolares, encontramos un porcentaje similar al de otras muestras españolas (Calmaestra, 2011; León et al., 2012) e inferior del que informan investigaciones internacionales (Smith et al., 2008). Por género, los resultados apuntan en la línea de otros estudios con una mayor presencia de chicas en el papel de cibervíctimas y de chicos como ciberagresores (Giménez et al., 2014; Ortega et al., 2008; Patchin e Hinduja, 2012; Tokunaga, 2010). Los análisis realizados indican además asociación significativa entre el consumo que los adolescentes hacen del teléfono móvil y del ordenador, y su implicación como agresores o agresores victimizados tanto en bullying como en cyberbullying.

La incidencia del bullying tradicional indica un porcentaje superior de adolescentes implicados respecto al cyberbullying, de nuevo con más chicas como víctimas y chicos como agresores, incidencia inferior a estudios previos realizados en el mismo contexto (Cerezo, 2001).

En relación con las conductas de uso problemático y posible adicción a las TIC, se encuentra que el trece por ciento de adolescentes murcianos muestran tales comportamientos, datos que superan a los encontrados en investigaciones previas (Carbonell et al., 2012; Kuss, Van Rooij, Shorter, Griffiths, y Van de Mheen, 2013), y que deberían ser tomados en consideración ante posibles problemas futuros de ciberadicción. El análisis por género señala algunas diferencias que interesa destacar: las chicas utilizan más el móvil y el ordenador que los chicos, muestran mayor malestar y ansiedad sin el móvil, sufren con más frecuencia cambios en sus ritmos de sueño e incluso manifiestan actitudes y reacciones agresivas cuando se les interrumpe durante la conexión a las TIC e internet; mientras que los chicos tienden más a recluirse en la habitación durante largos periodos de tiempo. Estas diferencias sugieren una mayor vulnerabilidad entre las chicas que entre los chicos. También conviene señalar que los estudiantes de educación secundaria se muestran más implicados en el uso problemático de las TIC que a sus compañeros de bachillerato, lo que implica un riesgo asociado a la edad, así pues a menor edad mayor posibilidad de adicción.

Se encuentra una asociación significativa entre las conductas de ciberadicción y la participación en bullying y cyberbullying. Destaca entre los implicados en bullying: descontrol de los ritmos del sueño y ansiedad por no usar el móvil. Entre los implicados en yberbullying destacan: discusión por el tiempo prolongado de uso de las TIC y sensación de malestar sin el móvil. La comparación entre ciberagresores y cibervíctimas apunta niveles más altos de comportamientos de uso problemático por parte de los que ejecutan la agresión. Entre las conductas concretas más notables, los ciberagreso- 
res modifican más sus ritmos de sueño y se molestan más cuando se les interrumpe la conexión a internet. Por consiguiente, se confirma que los implicados en tales dinámicas de acoso muestran niveles globales más altos de ciberadicción que los no implicados.

Los análisis referidos a la supervisión parental de los hijos durante su conexión a internet indican que escasamente uno de cada cuatro están controlados por algún adulto, a pesar de la influencia de la familia como factor de protección ante los riesgos de los menores con las TIC (Leung y Lee, 2012), como también ante conductas de riesgo social, consumo de drogas y agresión entre iguales (Cerezo y Méndez, 2012; Cerezo, Méndez y Ato, 2013). Entre los distintos miembros de la unidad familiar son fundamentalmente los padres los que supervisan a los hijos, seguidos de los hermanos mayores y, en menor medida los abuelos, existiendo además diferencias significativas por sexo que indican que son las hijas las más controladas, lo que indica la consabida percepción de una mayor fragilidad de las chicas, que demandaría una mayor protección familiar. Igualmente, se observa una declinación en las labores de supervisión a medida que aumenta la edad de los hijos, siendo los adolescentes de educación secundaria más controlados que los de bachillerato. Por otra parte, cabe destacar que las víctimas de bullying son más supervisadas durante la conexión a la red que los agresores. Sin embargo, no se aprecia asociación significativa entre el control parental durante la conexión a la red y la implicación en cyberbullying, lo que abunda en la soledad de la víctima, lo que viene subrayado por estudios como los de Leung y Lee (2012) y Vandebosch y Van Cleemput (2009), que destacan la importancia del control parental como pro-

\section{Referencias}

Álvarez, D., Núñez, J. C., Álvarez, L., Dobarro, A., Rodríguez, C. y González, P. (2011). Violencia a través de las tecnologías de la información y la comunicación en estudiantes de secundaria. Anales de Psicología, 27(1), 221-230.

Calmaestra, J. (2011). Cyberbullying: Prevalencia y características de un nuevo tipo de bullying indirecto. Tesis doctoral. Córdoba: Servicio de Publicaciones de la Universidad de Córdoba.

Cao, H., Sun, Y., Wan, Y., Hao, J., y Tao, F. (2011). Problematic Internet use in Chinese adolescents and its relation to psychosomatic symptoms and life satisfaction. BMC Public Health, 11(802), 1-8. doi: 10.1186/14712458-11-802

Carbonell, X., Fúster, H., Chamarro, A. y Oberst, U. (2012). Adicción a internet y al móvil: Una revisión de estudios empíricos españoles. Papeles del Psicólogo, 33(29), 82-89.

Cerezo, F. (2001). Variables de personalidad asociadas en la dinámica bullying (agresores versus víctimas) en niños y niñas de 10 a 15 años. Anales de Psicología, 17(1), 37-43.

Cerezo, F. y Méndez, I. (2012). Conductas de riesgo social y de salud en adolescentes. Propuesta de intervención contextualizada para un caso de bullying. Anales de Psicología, 28(3), 705-719.

Cerezo, F., Méndez, I. y Ato, M. (2013). Moderating role of family and friends' factors between disocial behavior and consumption in adolescents. International Journal of Clinical Health Psychology, 13, 171-180. doi.org/10.1016/S1697-2600(13)70021-8

Colás, P., González, T. y de Pablos, J. (2013). Juventud y redes sociales: Motivaciones y usos preferentes. Comunicar, 40 (XX), 15-23. doi: 10.3916/C40-201302-01 tección de los riesgos a los que se enfrentan los menores en la red, especialmente en cyberbullying. Esto nos lleva a plantear la necesidad de concienciar a los padres sobre la importancia de que supervisen a los hijos, incluyendo más tiempos de uso compartido de las tecnologías y coeducación para su uso responsable.

Las conclusiones de este trabajo permiten avanzar en el conocimiento del uso que los menores hacen de las TIC y, en concreto, de los peligros asociados al consumo de las mismas, su relación con experiencias de acoso tipo bullying y cyberbullying, y la necesidad de potenciar la supervisión familiar.

Entre las limitaciones del estudio, cabe señalar que se trata de un estudio descriptivo donde se recaba únicamente la información de los escolares. Sería de gran interés contrastarlos con la opinión de los adultos (profesorado y padres), así como indagar sobre las estrategias de supervisión familiar efectivas, que favorecen el uso responsable de las TIC. Por otro lado, aunque los análisis realizados permiten establecer inferencias a la población de escolares de la Región de Murcia sobre los diferentes objetivos aquí tratados, debe considerarse que el instrumento de recogida de información utilizado se basa en el autoinforme y esto presenta un sesgo de deseabilidad social que puede estar incidiendo en la baja tasa de implicados tanto de víctimas como de agresores en ambas dinámicas de acoso. Igualmente sería interesante, para futuras investigaciones, analizar de forma más exacta la frecuencia, extensión y duración con que se suceden tales situaciones lo que nos permitiría tener una visión más fiable del fenómeno y de su extensión entre la población escolarizada.

Devine, P. y Lloyd, K. (2012). Internet use and psychological wellbeing among 10year-old and 11-year-old children. Child Care in Practice, 18(1), 5-22. doi: 10.1080/13575279.2011.621888.

Echeburúa, E. y Corral, P. (2010). Adicción a las nuevas tecnologías y a las redes sociales en jóvenes: un nuevo reto. Adicciones, 22(2), 91-96.

Erdur-Baker, O. (2010). Cyberbullying and its correlation to traditional bullying, gender and frequent and risky usage of internet-mediated communication tools. New Media y Society, 12(1), 109-125. doi: $10.1177 / 1461444809341260$

Garaigordobil, M. (2011). Prevalencia y consecuencias del cyberbullying: una revisión. International Journal of Psychology and Psychological Therapy, 11(2), 233-254.

Garaigordobil, M. y Martínez, V. (2014). Efecto del Cyberprogram 2.0 sobre la reducción de la victimización y la mejora de la competencia social en la adolescencia. Revista de Psicodidáctica, 19(2), 289-305. doi: 10.1387/RevPsicodidact.10239.

Garmendia, M., Garitaonandia, C., Martínez, G. y Casado, M. A. (2012). Los menores en internet. Usos y seguridad desde una perspectiva europea. Quaderns del Cac, 38(1), 37-44.

Giménez, A. M., Maquilón, J. J. y Arnaiz, P. (2014). Acceso a las tecnologías, rendimiento y cyberbullying en escolares de educación secundaria. Revista Iberoamericana de Psicologia y Salud, 5(2), 119-133.

Gómes, F. y Sendín, J. C. (2014). Internet como refugio y escudo social: Usos problemáticos de la red por jóvenes españoles. Comunicar, 43(XXII), 45-53. doi: 10.3916/C43-2014-04

Kuss, D. J., van Rooij, A. J., Shorter, G. W., Griffiths, M. D. y van de Mheen, D. (2013). Internet addiction in adolescents: Prevalence and risk 
factors. Computers in Human Behavior, 29(5), 1987-1996. doi: 10.1016/j.chb.2013.04.002.

Labrador, F. J. y Villadangos, S. M. (2010). Menores y nuevas tecnologías: conductas indicadoras de posible problema de adicción. Psicothema, 22(2), 180-198.

Labrador, F. J., Villadangos, S. M., Crespo, M. y Becoña, E. (2013). Desarrollo y validación del cuestionario de uso problemático de nuevas tecnología (UPNT). Anales de Psicología, 29(3), 836-847.

León, B., Felipe, E., Fajardo, F. y Gómez, T. (2012). Cyberbullying en una muestra de estudiantes de Educación Secundaria: Variables moduladoras y redes sociales. Electronic Journal of Research in Educational Psychology, 10(2), 771-788.

Leung, L. y Lee, P. S. N. (2012). The influences of information literacy, internet addiction and parenting styles on internet risks. New media y Society, 14(1), 117-136. doi: 10-1177/1461444811410406.

Martínez, J. I., Cortés, A., Medrano, C. y Apodaca, P. (2014). Internet use and parental mediation: A cross-cultural study. Computers y Education, 70, 212-221. doi: 10.1016/j.compedu.2013.07.036.

Ministerio del Interior (2014). Encuesta sobre hábitos de uso y seguridad de internet de menores y jóvenes en España Recuperado de www.interior.gob.es/web/interior/

Ólafsson, K., Livingstone, S. y Haddon (2013). Children's Use of Online Technologies in Europe. A review of the European evidence base. LSE, London: EU Kids Online.

Ortega, R., Calmaestra, J. y Mora-Merchán, J. A. (2008). Estrategias de afrontamiento y sentimientos ante el cyberbullying. International Journal of Developmental and Educational Psychology, 1, 123-132.

Patchin, J. W. e Hinduja, S. (2012). Cyberbullying. An update and synthesis of the research. In J. W. Patchin y S. Hinduja (Eds.), Cyberbullying prevention and response. Expert perspectives (pp. 13-35). New York: Routledge.
Pedrero, E. J., Rodríguez, M. T. y Ruiz, J. M. (2012). Adicción o abuso del teléfono móvil. Revisión de la literatura. Adicciones, 24(2), 139-152.

Rial, A., Gómez, P., Braña, T. y Varela, J. (2014). Actitudes, perceordenadoriones y uso de Internet y las redes sociales entre los adolescentes de la comunidad gallega (España). Anales de Psicología, 30(2), 642-655.

Rodríguez, A. y Fernández, A. P. (2014). Relación entre el tiempo de uso de las redes sociales en internet y la salud mental en adolescentes colombianos. Acta Colombiana de Psicología, 17(1), 131-140. doi: 10.14718/ACP.2014.17.1.13

Schneider, S. K., O’Donnell, L., Stueve, A., y Coulter, R. W. S. (2012). Cyberbullying, school bullying, and psychological distress: A regional census of high school students. American Journal of Public Health, 102, 171-177.

Slonje, R., Smith, P. K. y Frisén, A. (2013). The nature of cyberbullying, and strategies for prevention. Computers in Human Behavior, 29, 26-32. doi: 10.1016/j.chb.2012.05.024

Smith, P. K., Mahdavi, J., Carvalho, M.., Fisher, S., Russell, S., y Tippett, N. (2008). Cyberbullying: its nature and impact in secondary school pupils. Journal of Child Psychology and Psychiatry, 49(4), 376-385. doi: 10.1111/j.1469-7610.2007.01846.x

Tokunaga, R. S. (2010). Following you home from school: A critical review and synthesis of research on cyberbullying victimization. Computers in Human Behavior, 26, 227-287. doi: 10.1016/j.chb.2009.11.014.

Vandebosch, H. y Van Cleemput, K. V. (2009). Cyberbullying among youngsters: profiles of bullies and victims. New Media y Society, 11(8), $1-$ 23. doi. $10.1177 / 1461444809341263$

Viñas, F. y González, M. (2010). Amenazas a través de la telefonía móvil e internet: perfil psicológico y consecuencias emocionales. Acción Psicológica, 7(1), 31-40.

(Articulo recibido: 15-01-2015; revisado: 17-05-2015; aceptado: 19-05-2015) 


\section{Anexo. Cuestionario Cyberbull}

Código del centro:

Género: $\square$ Chico $\square$ Chica

Curso:

Edad:

Nacionalidad: $\square$ Español/a $\square$ Otra

¿Participas en algún programa de apoyo educativo? $\square$ Sí

Si la respuesta es sí, señala con una cruz $(\mathrm{X})$ en cuál:

$\square$ Diversificación curricular $\square$ Compensación Educativa $\square$ Aula de apoyo $\square$ No lo sé

A continuación señala con una cruz $(\mathrm{X})$ la respuesta acorde con tu acceso y uso diario de las tecnologías y si has experimentado algunas de las situaciones por las que se pregunta. Marca máximo una respuesta por pregunta.

P1 ¿Tienes acceso a un teléfono móvil propio? $\square$ Sí $\square$ No

¿Tienes acceso a internet a través del móvil? $\square$ Sí $\square$ No

¿Cuántas horas al día sueles utilizar el teléfono móvil?

$\square$ Menos de $1 \mathrm{~h} \quad \square$ Entre 1 y $2 \mathrm{~h} \quad \square$ Entre 2 y $4 \mathrm{~h} \quad \square$ Más de $4 \mathrm{~h}$

P2 ¿Tienes acceso a un ordenador o portátil? $\square$ Sí $\square$ No

¿Tienes acceso a internet a través del ordenador? $\square$ Sí $\square$ No

¿Cuántas horas al día sueles utilizar el ordenador?

$\square$ Menos de $1 \mathrm{~h} \quad \square$ Entre 1 y $2 \mathrm{~h} \quad \square$ Entre 2 y $4 \mathrm{~h} \quad \square$ Más de $4 \mathrm{~h}$

P3. ¿Con qué frecuencia te pasan algunas de las siguientes situaciones? (Una cruz por cada ítem)

Me siento mal cuando no uso el móvil

Nunca Normalmente Casi siempre/ Siempre

Me siento mal cuando no uso el ordenador

Me enfado cuando me interrumpen mientras estoy con el móvil o el ordenador

Me acuesto tarde por estar con el móvil o el ordenador (sin estar estudiando)

Tengo necesidad constante de usar mi móvil

Tengo necesidad constante de usar el ordenador

Paso mucho tiempo en mi habitación con el móvil, consola o ordenador

Prefiero estar con el ordenador, móvil, TV que con mis amigos

Discuto con algún familiar por estar mucho tiempo con el móvil, consola, TV, ordena-

dor, etc.

P4. ¿'Te vigilan o controlan cuando estás conectado a internet? $\square$ Sí $\square$ No ¿Quién te vigila o controla en tu familia? (Una cruz por cada ítem)

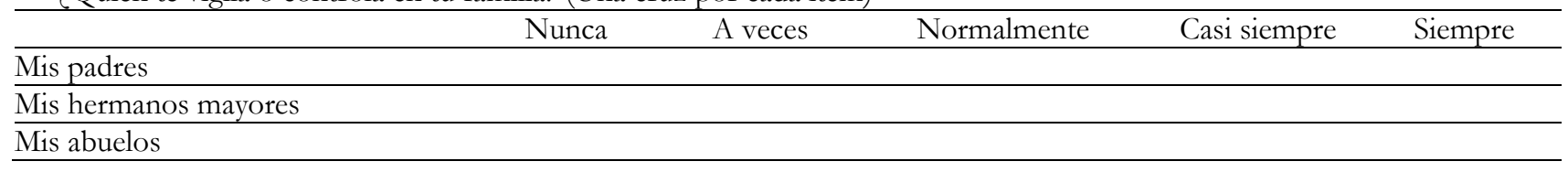

P5. ¿Has sufrido acoso de algún compañero/a en tu colegio o IES en el último mes? $\square$ Sí $\square$ No

P6. ¿Has acosado a algún compañero/a en tu colegio o IES en el último mes? $\square$ Sí $\square$ No

P7. ¿Has sufrido acoso de algún compañero/a a través del móvil o el ordenador? $\square$ Sí $\square$ No

P8. ¿Has acosado a otro compañero/a utilizando el móvil o el ordenador? $\square$ Sí $\square$ No 